\section{Heat shock proteins are no DAMPs, rather 'DAMPERs'}

\section{Femke Broere, Ruurd van der Zee and Willem van Eden}

Originally, the immune system was seen as a system that primarily combats infection. But, as discussed in the recent Review article by Grace Chen and Gabriel Nuñez (Sterile inflammation: sensing and reacting to damage. Nature Rev. Immunol. 10, 826-837 $(2010))^{1}$, most of us accept the idea that the immune system is key to tissue homeostasis and even to homeostatic interactions with the outside antigenic world, including the gut microbiota ${ }^{1,2}$. However, much remains unknown about the nature of the triggers of pro-inflammatory innate immune responses. In a broad sense, two main types of activators prevail: non-self antigens (known as pathogen-associated molecular patterns (PAMPs)), which are present in or released from infectious invaders; and damageassociated molecular patterns (DAMPs), which are host molecules (such as highmobility group box 1 protein (HMGB1)) released from damaged cells under necrotic but not apoptotic conditions.

Heat shock proteins (HSPs) are frequently mentioned as prime examples of DAMPs (see, for example, REFS 1,3). There are, however, several qualities inherent to HSPs that disqualify them as DAMPs.

First, DAMPs are intracellular molecules normally hidden from recognition by the immune system, whereas HSPs are freely present in the extracellular fluids ${ }^{4,5}$ and are also frequently exposed at the outer surface of cells (both eukaryotic and prokaryotic cells) ${ }^{6,7}$. Second, Toll-like receptor 2 (TLR2) and TLR4 are seen as two of the main receptors involved in the recognition of HSP60 and HSP70. These HSPs are released from cells under necrotic conditions, but it has been shown that TLR2 and TLR4 are not required for the host response to DAMPs that are derived from necrotic cells ${ }^{8}$. Moreover, other receptors for HSPs, such as the HSP70 scavenger receptor SRA1, have been shown to confer a suppressive rather than an activating signal to host cells ${ }^{9}$. Third, in vitro-cultured dendritic cells have been shown to adopt a tolerizing phenotype, rather than a mature or activated phenotype, in the presence of HSPs $^{10,11}$. Fourth, in experimental models of autoimmunity and of tissue or tumour transplants, immunization with HSPs was shown to lead to the induction of regulatory T cells, which suppressed disease or transplant rejection $^{12-19}$.

Taken together, these phenomena argue against the involvement of HSPs in the induction of the immune response to damagederived signals. On the contrary, HSPs seem to have a dampening effect on immune activation and have the capacity to promote immune homeostasis $^{12,20}$.

A possible reason for the proposition that HSPs are DAMPs could be that some of the early studies used recombinant HSP preparations that were contaminated with lipopolysaccharide (LPS), although in most cases contamination levels were not determined ${ }^{10,21}$. More recent studies (reviewed in REF. 5) using HSP preparations from which the contaminants had been effectively removed did not provide evidence to support a proinflammatory function for HSPs.

Based on the current experimental data, it seems that HSPs are key elements in the type of immune system responsiveness or reactivity that is induced by the following three typical conditions: one, sterile tissue damage, when tissue-derived HSPs in combination with DAMPs activate tissue repair and regulation; two, damage caused by infectious pathogens, when HSPs in the presence of DAMPs and pathogen-derived PAMPs lead to a full proinflammatory response with elimination of infection and regulation; and three, homeostatic interactions with commensal symbionts of the gut microbiota, when a combination of PAMPs and HSPs leads to the regulation and maintenance of symbiosis ${ }^{22-24}$. Given the ubiquitous presence and stressinducible nature of HSPs in both tissue cells and microbial invaders or symbionts, therapeutic targeting of HSPs offers an attractive possibility for the fine-tuning of such immune responses and the dampening of inflammation through the induction or activation of regulatory T cells.

Femke Broere, Ruurd van der Zee and Willem van Eden are at the Department of Infectious Diseases and Immunology, Faculty of Veterinary Medicine, University of Utrecht, Yalelaan 1, 3584CL Utrecht, The Netherlands.

Correspondence to W.v.E. e-mail:w.vaneden@uu.n! doi: $10.1038 /$ nri2873-c 1
Chen, G. Y. \& Nuñez, G. Sterile inflammation: sensing and reacting to damage. Nature Rev. Immunol. 10 826-837 (2010)

Lazzaro, B. P. \& Rolff, J. Danger, microbes, and homeostasis. Science 332, 43-44 (2011).

Wallin, R. P. A. et al. Heat-shock proteins as activators of the innate immune system. Trends Immunol. 23 130-135 (2002)

4. Mambula, S. S. et al. Mechanisms for HSP70 secretion: crossing membranes without a leader. Methods 43, 168-175 (2007).

5. Henderson, B. et al. Caught with their PAMPs down? The extracellular signaling actions of molecular chaperones are not due to microbial contaminants. Cell Stress Chaperones 15, 123-141 (2010).

6. Stangl, S. et al. Targeting membrane heat-shock protein 70 (Hsp70) on tumors by $\mathrm{cmHsp} 70.1$ antibody. Proc. Natl Acad. Sci. USA 108, 733-738 (2011).

7. Henderson, B. et al. Stress wars: the direct role of host and bacterial molecular chaperones in bacterial infection. Infect. Immun. 74, 3693-3706 (2006).

8. Chen, C. J. et al. Identification of a key pathway required for the sterile inflammatory response triggered by dying cells. Nature Med. 13, 851-856 (2007).

9. Wang, X. Y. et al. Scavenger receptor A negatively regulates antitumor immunity. Cancer Res. 67, 4996-5002 (2007).

10. Bendz, H. et al. Calcium signaling in DCs by human or mycobacterial HSP70 is caused by contamination and is not required for HSP70 mediated enhancement of cross-presentation. J. Biol. Chem. 39, 26477-26483 (2008).

11. Motta, A. et al. Mycobacterium tuberculosis HSP70 impairs maturation of DCs from bone marrow precursors, induces IL10 production and inhibits T cell proliferation in vitro. Immunology 121, 462-472 (2007).

12. van Eden, W. et al. Heat shock proteins induce $T$ cell regulation of chronic inflammation. Nature Rev. Immunol. 5, 318-330 (2005).

13. Wieten, L. et al. A novel heat-shock protein co-inducer boosts stress protein $\mathrm{Hsp} 70$ to activate T cell regulation of inflammation in autoimmune arthritis. Arthritis Rheum. 62, 1026-1035 (2010).

14. Tanaka, K.-I. et al. Genetic evidence for a protective role for heat shock factor 1 and HSP70 against colitis. J. Biol. Chem. 282, 23240-23252 (2007).

15. Kovalchin, J. T. et al. In vivo treatment of mice with heat shock protein gp96 improves survival of skin grafts with minor and major antigenic disparity. Transpl. Immunol. 15, 179-185 (2006).

16. Mirza, S. et al. The stress protein gp96 is not an activator of resting rat bone-marrow-derived dendritic cells, but is a co-stimulator and activator of CD3+ T cells. Cell Stress Chaperones 11, 364-378 (2006).

17. Slack, L. K. et al. Administration of stress proteins gp96 prolongs rat cardiac allograft survival, modifies rejection-associated inflammatory events and induces a state of peripheral T cell hyporesponsiveness. Cell Stress Chaperones 12, 71-82 (2007).

18. Borges, T. J. et al. Prolonged survival of allografts induced by mycobacterial HSP70 is dependent on CD $4{ }^{+} \mathrm{CD} 25+$ regulatory T cells. PLOS ONE 5, e 14264 (2010).

19. Chalmin, F. et al. Membrane associated HSP72 from tumor derived exosomes mediates STAT3-dependent immunosuppressive function of mouse and human myeloid-derived suppressor cells. J. Clin. Invest. 120 457-471 (2010).

20. Quintana, F. J. \& Cohen, I. R. The HSP60 immune system network. Trends Immunol. 32, 89-95 (2011).

21. Gao, B. \& Tsan, M. F. Endotoxin contamination in recombinant human HSP70 preparation is responsible for induction of TNF $\alpha$ release by murine macrophages. J. Biol. Chem. 278, 174-179 (2003).

22. Kojima, K. et al. Enteric flora and lymphocyte derived cytokines determine expression of heat shock proteins in mouse colonic epithelial cells. Gastroenterology 124, 1395-1407 (2003).

23. Hu, S. et al. Regional differences in colonic mucosaassociated microbiota determine the physiological expression of host heat shock proteins. Am. J. Physiol. Gastrointest. Liver Physiol. 299, G1266-G1275 (2010).

24. Rakoff-Nahoum, S. et al. Recognition of commensal microflora by Toll-like receptors is required for intestinal homeostasis. Cell 118, 229-241 (2004).

Competing interests statement

The authors declare no competing financial interests. 